\title{
Review: St John's Wort may be less effective than previously thought in people with depression
}

Werneke U, Horn O, Taylor DM. How Effective Is St. John's Wort? The Evidence revisited. J Clin Psychiatry 2004;65:611-17.

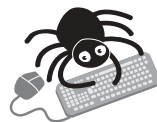

\section{METHODS}

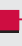

Design: Systematic review with meta-analysis.

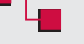

Data sources: Meta-analysis published in 2001 plus updated searches in MEDLINE and EMBASE (search date 2003) and hand searches of reference lists.

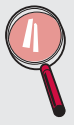

Study selection and analysis: Randomised controlled trials examining St John's Wort in people with depression, with participants diagnosed using the Hamilton Rating Scale for Depression (HAM-D) and using uniform criteria to measure treatment success (that is, 50\% reduction in baseline HAM-D score) were eligible. Exclusions: trials using St John's Wort in combination with other herbal preparations or to target psychiatric conditions other than depression. Two meta-analyses were conducted. One was a reproduction of the meta-analysis published in 2001 that included 15 studies (Meta-15). The other was an extension of the Meta-15 that included three subsequently published studies (Meta-18). Funnel plots, Begg's rank correlations, Egger's regressions, trim and fill methods, and meta-regressions were used to compare the Meta-15 and Meta- 18 results.

\section{MAIN RESULTS}

In both meta-analyses, St Johns's Wort was significantly more effective in reducing depressive symptoms compared with placebo, although St John's Wort was significantly more effective than placebo in the Meta-15 study compared with the Meta-18 study (see table 1). Univariate analysis showed that a decrease in sample size was associated with an increase in effect size for both Meta-15 and Meta-18 (see http://www.ebmentalhealth.com/supplemental for table 2). Publication in English was associated with a decrease in effect size for Meta-18, but this was insignificant in multivariate analysis. Effect size was weaker on multivariate analysis but remained significant.

Table 1 Comparison of the Meta-18 and Meta-15 St John's Wort studies

\begin{tabular}{lll}
\hline Study & RR $(95 \%$ Cl) & Adjusted RR* $(95 \%$ Cl) \\
\hline Meta-15 & $1.97(1.5$ to 2.5$)$ & $1.94(1.5$ to 2.5$)$ \\
Meta-18 & $1.73(1.4$ to 2.1$)$ & $1.30(1.0$ to 1.6$)$ \\
\hline
\end{tabular}

*Calculated using the trim and fill method, adding one study for Meta-15 and eight studies for Meta-18.

For correspondence: Ursula Werneke, Homerton Hospital, East Wing, Department of Psychiatry, Homerton Row, London, UK; Ursula.Werneke@ elcmht.nhs.uk

\section{CONCLUSIONS}

Although St John's Wort is effective for treating people with depression, adding three recent studies reduced the size of the effect.

\section{NOTES}

HAM-D baseline scores and placebo response were excluded as variables for regression models.

\section{Commentary}

Ceveral RCTs have shown St John's Wort (SJW) to be effective for mild to moderate depression. However, this is also the case with most of the antidepressants on the market. As there are over three dozen antidepressants, all claiming to be equally effective, it is a formidable task for the clinician to choose an antidepressant for a given patient. SJW is a relatively new addition to this long and ever growing list of antidepressants. Because of this confusion, there is a constant need to re-examine the evidence. The older meta-analyses have found SJW to be effective. However, in the meta-analysis by Werneke et al three recently published large studies have been included and as a result, SJW has been found to be less effective in the treatment of depression than previously published. This is a substantial new finding and is in accordance with the results of a recent large randomised controlled trial $^{1}$ that found SJW to be no better than placebo.

The finding of this study has important clinical implications. It shows that small studies overestimate effect size; therefore, the results could be misleading leading to premature conclusions that a drug is effective. SJW is an herbal remedy and is likely to be available in most countries "over the counter". There is a notion that herbal medicines are "safe" and "natural" without side effects and drug interactions: because of this widely held belief, SJW is likely to get prescribed more often than other antidepressants, however it is definitely not an innocuous medicine. ${ }^{2}$ There is a similar kind of belief about many Ayurvedic medicines which are basically herbal preparations. This study has succeeded in making a valid case for re-examination of the antidepressant property of SJW. Before concluding that SJW may be an ineffective drug we need to keep in mind that meta-analyses do not always agree among themselves ${ }^{3}$ and that the effect sizes of conventional antidepressants too are low. ${ }^{4}$ Therefore, large, simple trials also called "mega-trials" may be needed to further clarify the usefulness of SJW in treating depression. Dr Y C Janardhan Reddy DPM MD Associate Professor of Psychiatry, National Institute of Mental Health and Neurosciences (NIMHANS), Bangalore, INDIA

1 Hypericum Depression Trial Study Group. Effect of Hypericum perforatum (St. John's wort) in major depressive disorder: a randomized controlled trail. JAMA 2002;287:1807-14.

2 Swann AC. Commentary: St John's wort was not better than placebo for reducing symptoms in major depressive disorder. Evid Based Ment Health 2002;5:111.

3 Jadad AR, Cook DJ, Browman GP. A guide to interpreting discordant systematic reviews. Can Med Assoc J 1997; 156:141 1-16.

4 Bech P, Ciadella P, Haugh MC, et al. Meta-analysis of randomized controlled trials of fluoxetine $v$. placebo and tricyclic antidepressants in short term treatment of major depression. Br J Psychiatry 2000;176:421-8.

Please see http://www.ebmentalhealth.com/supplemental for additional commentary by Professor Pinarosa Avato. 